\title{
Propriedades Mecânicas e Morfologia de uma Blenda Polimérica de PP/HIPS Compatibilizada com SEBS
}

\author{
Tomás J. A. de Mélo e Laura H. Carvalho \\ Departamento de Engenharia de Materiais / UFPB
}

Ricardo B. Calumby e Keila G. Q. Brito

Engenharia de Materiais / UFPB

J osé R. M. D'Almeida

Departamento de Ciência dos Materiais e Metalurgia, PUC/RJ

\section{E. Spieth \\ Interconsult, Salvador, BA}

Resumo: Neste trabalho o efeito da adição de 2 a $7 \%$ em peso de um copolímero tribloco estireno-b-etilenoco-butileno-b-estireno (SEBS) em uma blenda polipropileno/poliestireno de alto impacto (PP/HIPS) 70:30 foi determinado. As propriedades em tração e impacto, temperatura de amolecimento Vicat e morfologia da blenda PP/HIPS (70:30) em função da concentração de SEBS foram determinadas. As blendas foram processadas em extrusora dupla-rosca contra-rotativa interpenetrante e as amostras moldadas por compressão. Os resultados mostraram que, embora a adição do SEBS tenha promovido um aumento muito leve na resistência tênsil e no módulo elástico, observou-se um expressivo aumento no alongamento na ruptura e na resistência ao impacto das blendas; a temperatura Vicat das blendas foi superior à do HIPS e que a adição do SEBS promoveu uma redução significativa do tamanho de partícula, indicando que o SEBS exerceu um efeito emulsificante e estabilizador na morfologia das blendas investigadas. Resultados otimizados foram obtidos para a composição contendo 5\% de SEBS, o que foi atribuido à uma maior compatibilidade entre as fases.

Palavras-chave: Blenda polimérica, compatibilizante, polipropileno, poliestireno de alto impacto, SEBS, HIPS.

\section{Mechanical Properties and Morphology of a PP/HIPS Polymer Blend Compatibilized with SEBS}

In this work the effect of a 2-7\% w/w styrene-b-ethylene-co-butylene-b-styrene triblock copolymer (SEBS) addition on the properties of a 70:30 polypropylene/high impact polystyrene (PP/HIPS) polymer blend was investigated. Tensile and impact properties, the Vicat temperature and blend morphology were determined as a function of SEBS content. Processing was carried out in a twin-screw intermeshing counter-rotating extruder and the samples were compression moulded. Our results indicate that the SEBS addition, albeit promoting very slight increases on tensile strenght and modulus, led to increases on the elongation at break and impact strenght of the blends, and the Vicat temperature of the blends was higher than that of HIPS. The SEM analysis showed that SEBS addition promotes a significant reduction on particle size, which was taken as an indication that SEBS exerted an emulsifying and stabilizing effect on the morphology of the PP/HIPS blend under investigation. The best overall properties were obtained at $5 \mathrm{wt} . \%$ SEBS content.

Keywords: Polymer blend, compatibiliser, polypropylene, high impact polystyrene, SEBS, HIPS.

Autor para correspondência: Laura H. Carvalho, Depto de Engenharia de Materiais, CCT, Universidade Federal da Paraíba, C. P. 10034, CEP: 58109-970, Campina Grande, PB. E-mail: laura@dema.ufpb.br 


\section{Introdução}

O polipropileno é um polímero versátil e de larga aplicação, porém apresenta instabilidade dimensional e limitações na resistência ao impacto e termoformabilidade ${ }^{[1]}$. Por outro lado, quando modificado com borracha ou misturado com outros polímeros, suas propriedades são otimizadas podendo ser aplicado em parachoques de automóveis, revestimento interno de freezers, eletrodomésticos, etc ${ }^{[1,2]}$. Sistemas compostos por polipropileno e poliestireno ou poliestireno de alto impacto (HIPS), são imiscíveis, resultando em segregação de fases e compostos com propriedades insuficientes ${ }^{[1-6]}$. Portanto, para melhorar o desempenho deste tipo de composto, é necessário promover a interação interfacial entre as fases presentes, o que em geral é feito pela adição de um agente compatibilizante ${ }^{[7,8]}$. Esses agentes são copolímeros em bloco, de enxertia (graft copolymers), funcionais ou reativos, que possuem estruturas quimicamente semelhantes, ou com grupos químicos reativos, aos componentes da blenda ${ }^{[9]}$. Geralmente, os copolímeros tendem a segregar na interface entre as fases da blenda e seus segmentos de cadeia mostram-se capazes de penetrar nas respectivas fases do sistema $^{[8,9]}$. Um dos efeitos do compatibilizante é reduzir a tensão interfacial entre a fase dispersa e a matriz, ou seja, apresentar um efeito emulsificante, facilitando a dispersão de uma fase na outra. Outro efeito é aumentar a interação, e portanto a adesão, entre os contornos das fases facilitando a transferência de tensões e, um terceiro efeito, é estabilizar a fase dispersa evitando a sua coalescência ${ }^{[10]}$. Na prática, todos esses efeitos tendem a ocorrer e, além disto, promovem mudanças no comportamento reológico da blenda ${ }^{[7-9]}$. A morfologia final de um sistema está relacionada com o comportamento reológico, composição, a razão de viscosidade e elasticidade dos componentes, com a tensão interfacial e a taxa e tensão de cisalhamento, desenvolvidas no processo de mistura e/ou de transformação ${ }^{[8,9]}$. Portanto, esses fatores determinarão qual das fases será a dispersa e qual será a contínua ${ }^{[8,9]}$. A morfologia da fase dispersa em sistemas poliméricos imiscíveis pode se apresentar na forma de gotículas ("droplets"), ou ainda na forma de corpos cilíndricos ou fibrilares ("threads"). Misturas binárias de PP com SEBS ${ }^{[11-14]}$ revelaram que a adição do SEBS, em geral, leva a um decréscimo na tensão no escoamento e módulo de elasticida- de e ao mesmo tempo provoca um aumento na resistência ao impacto e um melhoramento nas propriedades reológicas e fratura do fundido, o que é desejável quando se quer melhorar a processabilidade de um sistema. Diversas misturas de PP/PS, PP/HIPS, PP/ HDPE, PP/PC, PP/PEBD têm apresentado resultados interessantes quando compatibilizados com copolímeros EPR, $\mathrm{SBR}^{[1]}$ e principalmente com SEBS ${ }^{[2,11-17]}$. Esses sistemas podem combinar a rigidez, resistência ao risco e excelente resistência ao fissuramento sob tensões ambientais ("enviromental stress cracking") do polipropileno, com a tenacidade, termoformalibilidade e/ou rigidez do segundo componente, que em geral está em menor proporção na composição.

Neste trabalho a eficiência do copolímero tribloco estireno-b-etileno-co-butileno-b-estireno (SEBS), como agente compatibilizante para uma blenda polimérica polipropileno/poliestireno de alto impacto PP/HIPS (70:30), foi avaliada. O SEBS utilizado possui razão de estireno/borracha (71/29) e foi adicionado em concentrações de 2 a $7 \%$ em peso. A composição da blenda foi escolhida mediante otimização experimental, realizada em estudos anteriores em misturadores contínuos distintos, com o objetivo de obter o melhor balanço possível entre rigidez e impacto do sistema ${ }^{[2]}$. As blendas, com e sem agente compatibilizante, foram processadas em extrusora dupla-rosca e as amostras moldadas por compressão. Avaliou-se as resistências à tração e ao impacto dos sistemas, bem como a sua temperatura de amolecimento Vicat e a morfologia das misturas em função da concentração do compatibilizante SEBS.

\section{Experimental}

\section{Materiais}

Os materiais empregados neste trabalho foram o polipropileno da linha Suplen da Polibrasil S/A Indústria e Comércio, com índice de fluidez de $2,5 \mathrm{~g} /$ 10 min e o poliestireno de alto impacto da linha 477 da EDN-Estireno do Nordeste S/A, com índice de fluidez de 4,8 g/10min. Como agente compatibilizante foi usado o copolímero tribloco SEBS, com razão estireno/borracha (71/29), marca Kraton ${ }^{\circledR}$ G $1652^{[5]}$, cedido pela Shell Química Corporation.

Os valores de índice de fuidez (IF) foram obtidos em plastômetro DSM MI-3, de acordo com a norma 
ASTM D 1238. Os resultados reportados referem-se à uma média de 5 testes por composição.

\section{M etodologia}

As blendas PP/HIPS (70:30) sem compatibilizante e com teores de agente compatibilizante de $2 ; 3,5 ; 5$ e $7 \%$ em peso, foram misturadas a seco por tamboreamento durante 15 minutos. Posteriormente foi realizada uma mistura intensiva à quente em extrusora dupla-rosca com razão $\mathrm{L} / \mathrm{D}=25$, acoplada a um reômetro de torque System 90 da Haake-Blucher. As seguintes condições de mistura foram empregadas: perfil de temperatura $160 / 190 / 210 / 220 / 220^{\circ} \mathrm{C}$, velocidade de rotação de rosca de $50 \mathrm{rpm}$ e alimentação constante. Os extrusados, depois de resfriados naturalmente à temperatura ambiente $\left(\sim 25^{\circ} \mathrm{C}\right)$, foram granulados em um moinho de facas e moldados por compressão (prensa de marca SCHULZ) na temperatura de $190 \pm 5^{\circ} \mathrm{C}$ e sob uma pressão de 9,81 $\mathrm{MPa}$, na forma de corpos de prova de tração e impacto, segundo as normas ASTM (especificadas nos ítens abaixo). Os dados apresentados foram obtidos de uma média de 10 corpos de prova. O componentes de partida PP e HIPS foram submetidos à mesma história termo-mecânica das blendas.

\section{Caracterização Mecânica}

Os testes de resistência à tração foram realizados em Máquina Universal de Ensaios Testometric Micro 350 com capacidade de $500 \mathrm{Kg}$, operando segundo a norma ASTM D-638. Utilizou-se corpos de prova do tipo I e velocidade de ensaio de $50 \mathrm{~mm} / \mathrm{min}$.

Os testes de resistência ao impacto Izod, foram conduzidos em uma máquina Zwick 5101/100 segundo a norma ASTM D 256. Os corpos de prova foram entalhados, em um entalhador CEAST 6525/000, com dimensões nominais de $75 \times 12 \times 3 \mathrm{~mm}$ e entalhe de $1 \mathrm{~mm}$ de profundidade, e submetidos à fratura com pêndulo de $4,0 \mathrm{~J}$ à temperatura ambiente $\left(\sim 25^{\circ} \mathrm{C}\right)$ (único pêndulo disponível para o ensaio). A energia por unidade de largura da amostra foi registrada.

\section{Caracterização Termo-M ecânica}

$\mathrm{O}$ ensaio de temperatura de amolecimento Vicat foi realizado, segundo a norma ASTM D 1525, empregando-se uma taxa de aquecimento de $120^{\circ} \mathrm{C} / \mathrm{h}$. Os dados reportados na Tabela 1 foram obtidos de uma média de três medições. Embora não seja um ensaio típico para sistemas multifásicos, o teste foi utilizado qualitativamente como parâmetro comparativo.

\section{Caracterização M orfológica}

A análise morfológica foi realizada em Microscópio Eletrônico de Varredura (MEV), Zeiss DSM 960, operando no modo de elétrons secundários, com aceleração do feixe entre 15 e $20 \mathrm{kV}$ e corrente de $80 \mu \mathrm{A}$. Analisou-se a superfície de fratura dos corpos de prova entalhados e submetidos ao impacto. Os resultados obtidos foram utilizados na avaliação do efeito da composição do agente compatibilizante SEBS na morfologia das blendas de PP/HIPS.

\section{Resultados e Discussão}

\section{Caracterização Mecânica}

A Tabela 1 mostra os resultados das propriedades obtidas em ensaios sob tração, impacto e temperatura Vicat, avaliando o comportamento dos componentes individualmente e das blendas PP/HIPS (70:30), com $0-7 \%$ em peso do copolímero SEBS. Observase que a blenda PP/HIPS (70:30) sem compatibilizante apresentou, para todas as propriedades avaliadas, valores inferiores aos dos componentes individuais da

Tabela 1. Propriedades mecânicas das blendas PP/HIPS em função do teor de SEBS.

\begin{tabular}{cccccc}
\hline Composição & $\boldsymbol{\sigma}_{\mathbf{y}}(\mathbf{M P a})$ & $\boldsymbol{\varepsilon}_{\mathbf{r}}(\boldsymbol{\%})$ & $\mathbf{E}(\mathbf{G P a})$ & $\mathbf{R . I}_{\text {Izod }}(\mathbf{J} / \mathbf{m})$ & $\mathbf{T}_{\text {VICAT }}\left({ }^{\circ} \mathbf{C}\right)$ \\
\hline PP & $30,5 \pm 0,3$ & $332,5 \pm 38$ & $0,8 \pm 0,0$ & $18,3 \pm 0,6$ & $147,5 \pm 0,7$ \\
HIPS & $23,6 \pm 0,8$ & $14,7 \pm 5,9$ & $1,0 \pm 0,1$ & $32,8 \pm 0,6$ & $103,0 \pm 1,4$ \\
$70 / 30$ & $22,1 \pm 1,1$ & $13,9 \pm 4,0$ & $0,5 \pm 0,0$ & $9,8 \pm 0,1$ & $136,0 \pm 1,4$ \\
$70 / 30 / 2$ & $24,7 \pm 1,5$ & $9,5 \pm 2,1$ & $0,7 \pm 0,1$ & $16,8 \pm 1,4$ & $134,0 \pm 1,4$ \\
$70 / 30 / 3,5$ & $24,7 \pm 0,8$ & $11,8 \pm 3,9$ & $0,8 \pm 0,1$ & $21,3 \pm 1,9$ & $130,5 \pm 0,7$ \\
$70 / 30 / 5$ & $26,5 \pm 1,0$ & $27,5 \pm 5,8$ & $0,8 \pm 0,0$ & $31,8 \pm 2,2$ & $141,0 \pm 1,4$ \\
$70 / 30 / 7$ & $22,0 \pm 0,3$ & $25,7 \pm 4,8$ & $0,6 \pm 0,0$ & $26,7 \pm 4,2$ & $127,0 \pm 0,0$ \\
\hline
\end{tabular}


mistura. Estes resultados demonstram a incompatibilidade do sistema PP/HIPS, conforme já reportado em trabalho anterior ${ }^{[2]}$. Para facilitar a discussão e visualização, os dados da Tabela 1 estão apresentados graficamente nas Figuras 1 a 5 .

As Figuras 1 e 2, mostram que a adição de até 5\% do copolímero SEBS, promoveu um discreto aumento no limite de escoamento, $\sigma_{\mathrm{y}}$ e no módulo elástico, E, da blenda PP/HIPS (70:30), enquanto no sistema com 7\% estes valores praticamente não foram alterados. $\mathrm{O}$ aumento nestas propriedades é tomado como um indicativo que a adição de SEBS promoveu a interação entre as fases presentes, fortalecendo a interface do sistema, o que é fundamental para a transferência de tensões entre as fases. A falta desta interação acarreta a presença de microvazios nas interfaces, o que leva à formação de trincas, e facilita a propagação destas, resultando em baixa resistência ${ }^{[9]}$. Mudanças ocorridas na morfologia, assim como evidências de partículas rompidas no plano de fratura, conforme será discutido posteriormente, indicam a ocorrência de adesão interfacial. Observa-se

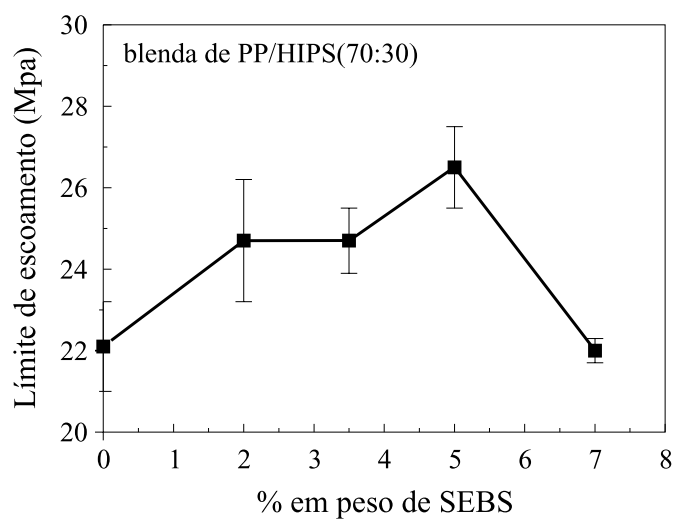

Figura 1. Limite de escoamento das blendas PP/HIPS (70:30) com 0-7\% em peso do copolímero SEBS.

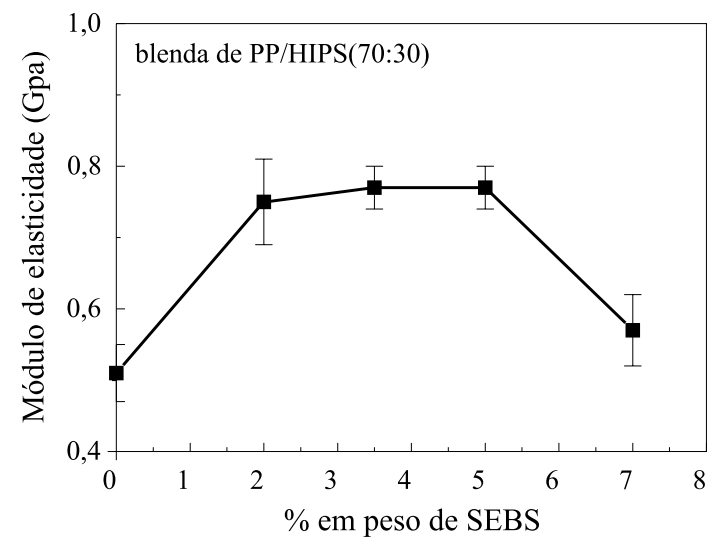

Figura 2. Módulo elástico das blendas PP/HIPS (70:30) com 0-7\% em peso do copolímero SEBS. ainda nas Figuras 1 e 2 que tanto o limite de escoamento quanto o módulo elástico apresentaram um máximo no sistema com 5\% de SEBS, indicando que na faixa de composições investigada, esta concentração otimiza a rigidez da blenda PP/HIPS.

As Figuras 3 e 4 mostram que o alongamento na ruptura e a resistência ao impacto, foram aumentados com a introdução de SEBS. Observa-se que o melhor resultado de resistência ao impacto foi obtido novamente com a adição de $5 \%$ de SEBS. Nesse caso, dentro da margem de erro experimental, obteve-se propriedades mecânicas à tração e impacto comparáveis às do HIPS comercial, que foi processado e moldado em condições idênticas às das blendas. Pode-se observar, ainda, que a resistência ao impacto da formulação com 5\% de SEBS é cerca de $220 \%$ superior à resistência ao impacto da blenda sem compatibilizante (Tabela 1). Estes dados fortalecem as suposições que o SEBS atua como um agente compatibilizante neste sistema. A compatibilização pode ser atribuída à difusão do SEBS para as

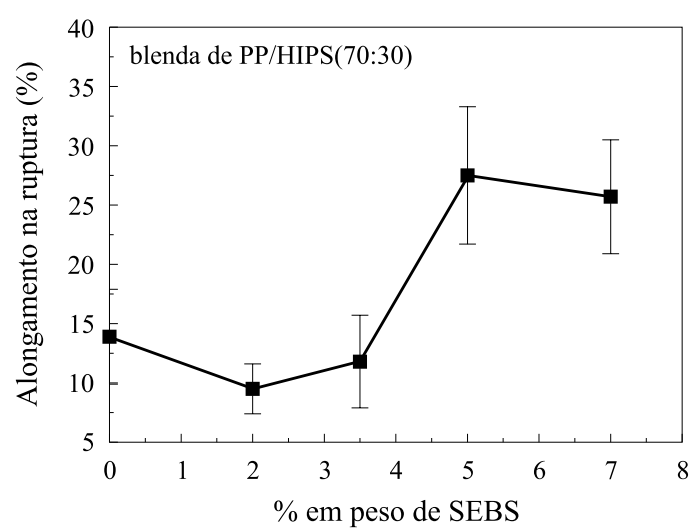

Figura 3. Alongamento na ruptura das blendas PP/HIPS (70:30) com 0-7\% em peso do copolímero SEBS.

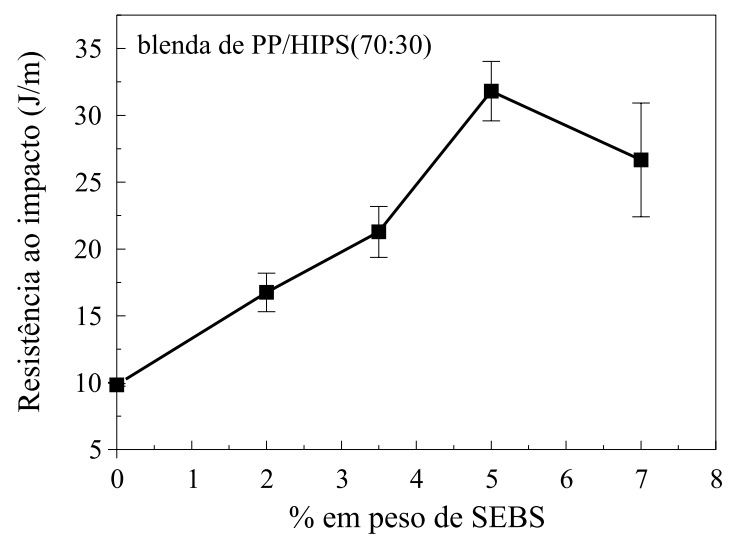

Figura 4. Resistência ao impacto das blendas PP/HIPS (70/30) com 0-7\% em peso do copolímero SEBS. 
interfaces, promovendo maior interação entre os segmentos moleculares estirênicos e olefínicos do compatibilizante com os da blenda PP/HIPS durante a mistura mecânica dos componentes, conforme reportado por vários autores ${ }^{[13-15,18]}$. Segundo alguns autore ${ }^{[15,19]} \mathrm{o}$ aumento do alongamento na ruptura $\mathrm{e}$ da resistência ao impacto estão relacionados com os processos de dissipação de energia, tais como microfibrilamento sob tensão e microescoamento sob cisalhamento. O primeiro mecanismo ocorre preferencialmente em matrizes frágeis, como o PS, ou tenacificadas, como o HIPS ${ }^{[22]}$. O segundo mecanismo ocorre em matrizes dúcteis, como o PP ${ }^{[22]}$. Porém, em sistemas multifásicos como o estudado neste trabalho, estes mecanismos podem atuar simultaneamente, em uma interação complexa. Os resultados obtidos, em geral, demonstram que o copolímero SEBS proporciona um aumento na rigidez da blenda PP/HIPS (70:30) sem comprometer significativamente a resistência ao impacto, o que é surpreendente quando comparado a outros sistemas apresentados na literatura $^{[5,13-15,20,21]}$.

\section{Caracterização Termo-M ecânica}

Embora o ensaio de temperatura de amolecimento Vicat não seja o mais adequado para sistemas multifásicos ${ }^{[22]}$, o mais usual é o ensaio de HDT ${ }^{[22]}$, este ensaio foi utilizado como um indicativo qualitativo do comportamento térmico do sistema quando submetido a uma carga e taxa de aquecimento constantes. As condições de teste foram as recomendadas pela norma ASTM D 1525. Na Figura 5 e na Tabela 1, observa-se que a adição do compatibilizante SEBS reduz a temperatura de amolecimento Vicat para todas as composições à exceção da formulação com 5\% de SEBS. Em termos comparativos, a temperatura Vicat das blendas, com e sem agente compatibilizante, foi, em média, $30^{\circ} \mathrm{C}$ superior à do HIPS, sendo que para a blenda com $5 \%$ de SEBS, o aumento foi de quase $40^{\circ} \mathrm{C}$. A maior temperatura de amolecimento das blendas em relação ao HIPS foi atribuída a presença do PP na composição das blendas, já que o PP possui temperatura de amolecimento Vicat superior à do HIPS. Portanto, como esperado, os sistemas apresentaram temperaturas intermediárias entre o PP e o HIPS. Por outro lado, o sistema com 5\% de SEBS mostrou um comportamento diferenciado dos outros, o que indica que a presença do SEBS nesta concentração tornou o sistema mais rígido. Os dados referentes ao limite de escoamento e ao módulo elástico,

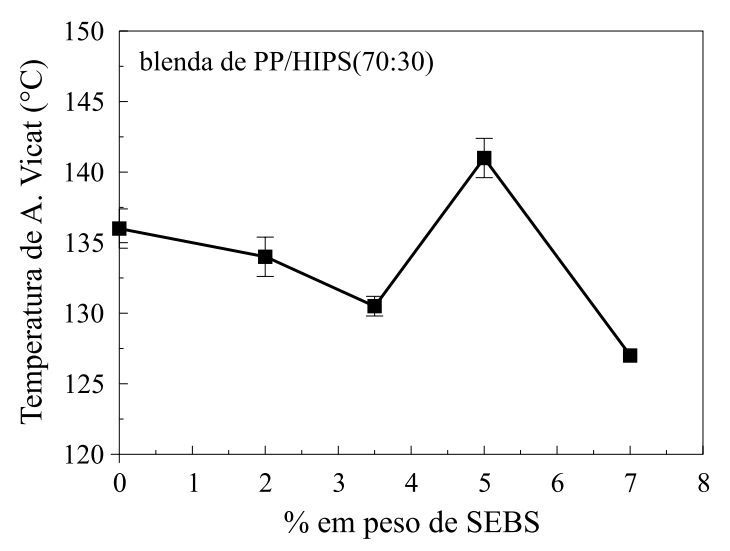

Figura 5. Temperatura de amolecimento Vicat das blendas PP/HIPS (70:30) com 0-7\% em peso do copolímero SEBS.

bem como o aspecto morfológico apresentado a seguir, corroboram esta suposição.

\section{Caracterização M orfológica}

As Figuras 6 a 10 mostram a superfície de fratura sob impacto Izod, realizado à temperatura ambiente, da blenda PP/HIPS (70:30) sem e com a adição de 2-7 \% em peso do compatibilizante SEBS. As superfícies de fratura analisadas não foram atacadas com nenhum solvente. Observa-se na Figura 6, para o sistema sem compatibilizante, a formação de uma estrutura bifásica com partículas esféricas ou arredondadas de HIPS, de tamanhos variados e muito próximas, dispersas na matriz de PP. Na literatura, observou-se morfologia parecida utilizando o sistema PP/PS ${ }^{[1,3,6,15]}$. Diversos estudos mostram que o tamanho, a forma e a distribuição dos domínios da fase discreta, são resultantes de efeitos viscosos do fundido ou efeitos viscoelásticos, equilibrados pela tensão interfacial e condições de mistura ${ }^{[7-9,18,23-26]}$. Embora não seja objetivo deste trabalho enfatizar a influência de tamanho, forma e distribuição de tamanhos de partículas, sabe-se, entretanto, da sua importância nos mecanismos de microdeformação que se reflete nas propriedades finais da blenda ${ }^{[8,19]}$.

Na Figura 6 podem ser observados espaços vazios entre as fases (setas brancas), o que indica uma pobre adesão entre a fase dispersa e a fase contínua, caracterizando um sistema incompatível. A má adesão ocorre devido a alta tensão interfacial existente entre os componente ${ }^{[12,15]}$, o que torna a interface com baixa resistência. De fato, como pode ser verificado na Figura 6, várias partículas foram sacadas totalmente da matriz quando da fratura do corpo de prova. A incompatibilidade entre a matriz e a fase dispersa, visualizada através da morfologia da superfície de fratura, corrobora 


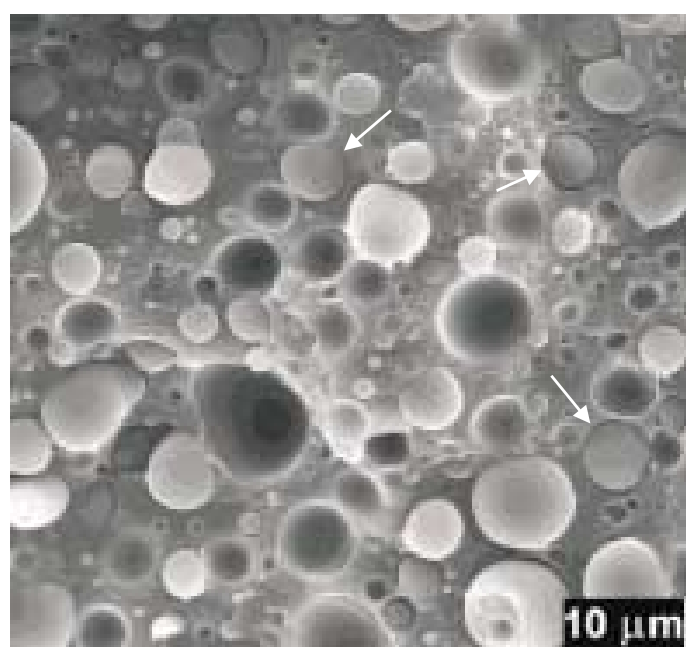

Figura 6. Morfologia de uma blenda PP/HIPS (70:30) sem o SEBS. (setas brancas indicam a fraca adesão). Aumento: $1000 \mathrm{X}$.

os resultados das propriedades mecânicas desse sistema, que foram inferiores às dos sistemas compatibilizados (ver Figuras 1-5 ou Tabela 1).

As Figuras 7 a 10 mostram as superfícies de fratura de amostras de PP/HIPS (70:30) compatibilizadas com 2; 3,5; 5 e $7 \%$ em peso de SEBS, respectivamente. Pode ser observado claramente que ocorreu uma elevada redução no tamanho das partículas dispersas na matriz, principalmente com teores de SEBS entre $2-5 \%$ em peso. Esse efeito pode acontecer devido a difusão e habilidade do compatibilizante em permanecer na interface entre as fases dos constituintes, promovendo uma redução da energia interfacial e evitando o coalescimento das partículas ${ }^{[10]}$. A redução no tamanho da fase discreta dentro de certo limite ${ }^{[19]}$ é importante no resultado final, pois aumenta a área interfacial e melhora a transferência de tensões impostas ao sistema. Verifica-se também, que a adição do compatibilizante promoveu a adesão interfacial, elevando a resistência mecânica da blenda, como discutido anteriormente. Na Figura 9, está mostrado (indicação com seta branca) que a adesão entre as fases foi boa, pois observa-se partículas esféricas rompidas no plano, evidenciando que a interface não rompeu e confirmando que a adesão entre as fases foi aumentada em relação ao sistema sem compatibilizante. É importante notar nesta figura que é um pouco difícil distinguir a fase dispersa HIPS na matriz de PP, ou de SEBS na matriz PP, e que seria necessário remover, por dissolução, uma das fases para a melhor compreensão e identificação dos constituintes. A Figura 11, exibe em maior aumento, a superfície de fratura da blenda com 5\% de SEBS, onde observa-se

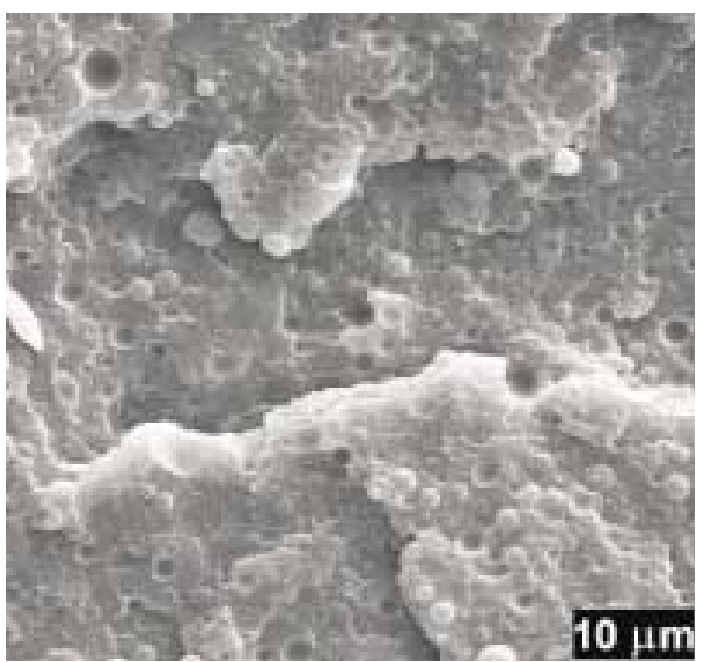

Figura 7. Morfologia de uma blenda PP/HIPS (70:30) com 2\% de SEBS. Aumento: $1000 \mathrm{X}$.

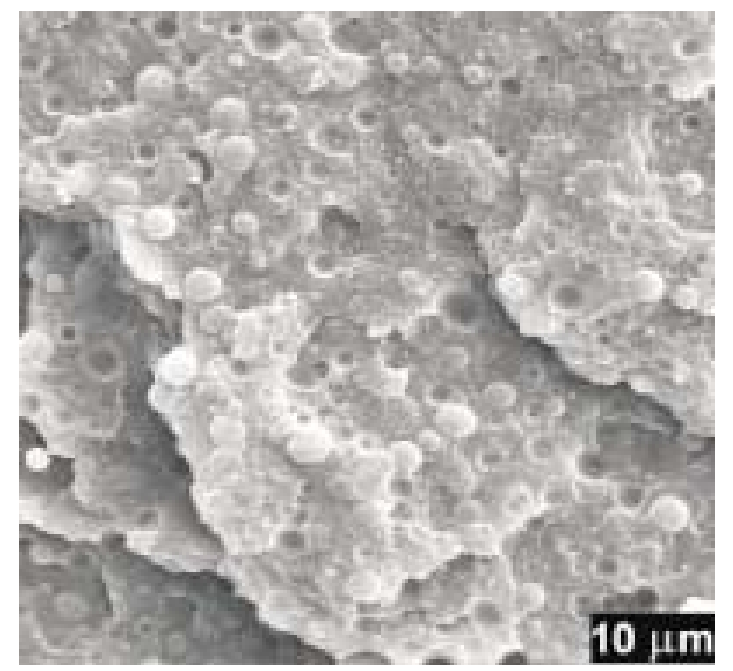

Figura 8. Morfologia de uma blenda PP/HIPS (70:30) com 3,5\% de SEBS. Aumento: $1000 \mathrm{X}$.

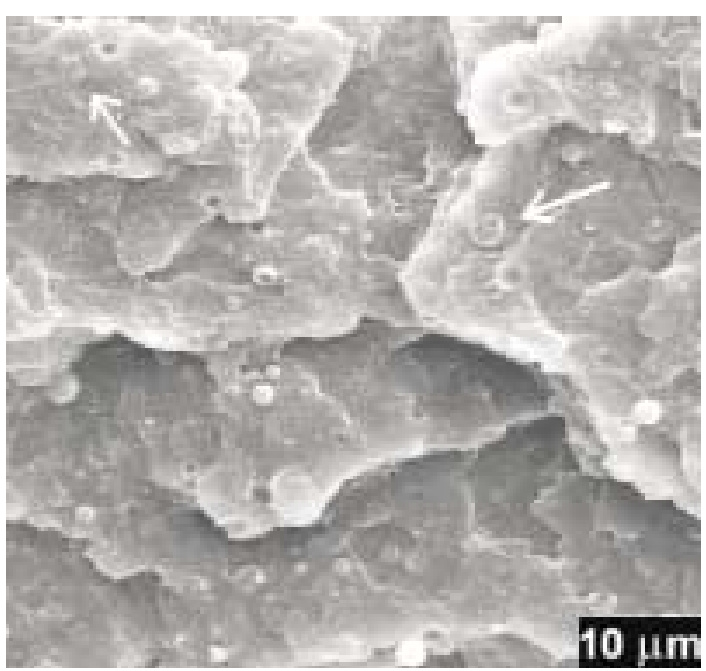

Figura 9. Morfologia de uma blenda PP/HIPS (70:30) com 5\% de SEBS. Aumento: $1000 \mathrm{X}$. 
com mais nitidez a ruptura de partículas no plano, sem rompimento da interface.

Observa-se que a adição de SEBS levou a uma redução no tamanho e a uma melhor dispersão das partículas no sistema PP/HIPS. Verificou-se ainda que, em teores de 5\% de SEBS, houve também uma melhor adesão entre as fases, como pode ser observado comparativamente nas micrografias das Figuras 7-10. Esta hipótese esta melhor evidenciada na Figura 11, onde pequenas partículas dispersas foram rompidas no plano de propagação de fratura, apresentando melhor adesão com a matriz de PP. Já o sistema PP/HIPS com $7 \%$ de SEBS, Figura 10, apresentou em relação aos demais sistemas, uma morfologia onde as partículas discretas aparentam estar em maior quantidade e consequentemente mais próximas, além de revelar um grande números de partículas sacadas na superfície de fratura do corpo de prova. Nesta concentração, o compatibilizante mostrou-se menos eficaz em relação a rigidez, como pode ser verificado através dos valores do limite de escoamento e módulo elástico. Porém, o alongamento na ruptura e a resistência ao impacto, foram superiores aos das blendas com $2 \mathrm{e}$ 3,5\% em peso de SEBS, caracterizando a ocorrência de adesão. Entretanto, para uma discussão maior sobre os aspectos morfológicos obtidos, será necessária uma investigação mais detalhada, por exemplo, através de ataque químico e remoção de fase, para poder-se identificar com maior precisão na superfície de fratura onde localizam-se predominantemente os domínios da fase dispersa HIPS e da fase elastomérica

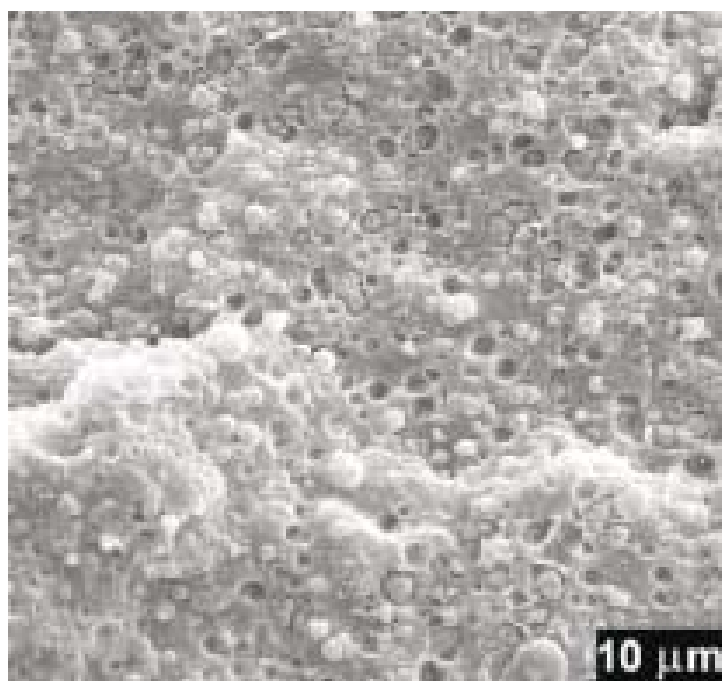

Figura 10. Morfologia de uma blenda PP/HIPS (70:30) com 7\% de SEBS. Aumento: $1000 \mathrm{X}$.
SEBS, conforme comentado anteriormente e verificado em outros sistemas ${ }^{[1,13-15]}$.

\section{Conclusões}

Neste trabalho avaliou-se o efeito compatibilizante do copolímero SEBS nas propriedades mecânicas e na morfologia de blenda PP/HIPS. Observou-se que a incorporação do SEBS em pequenas quantidades (2-7\% em peso) elevou discretamente a rigidez da blenda e aumentou significativamente $\mathrm{o}$ alongamento na ruptura e a resistência ao impacto. A temperatura de amolecimento Vicat foi reduzida com a adição de SEBS, exceto para blenda com $5 \%$ em peso. Valores otimizados do sistema PP/HIPS estudado foram obtidos empregando-se 5\% em peso do compatibilizante. Esta composição apresentou, dentro do erro experimental, propriedades semelhantes às do HIPS comercial.

A morfologia da blenda PP/HIPS revelou-se com uma fase contínua de PP e outra fase de partículas discretas de HIPS com aparência esférica. A interface entre as fases apresentou vazios, caracterizando uma pobre adesão. Entretanto, com a introdução de SEBS, ocorreu uma redução drástica no tamanho dos domínios discretos, sendo este efeito mais evidente nas composições com 2-5\% de SEBS. Verificou-se na superfície fraturada, partículas rompidas no plano, caracterizando adesão entre fases. As variações encontradas nas propriedades mecânicas em função da composição das blendas foram consistentes com as alterações morfológicas observadas.

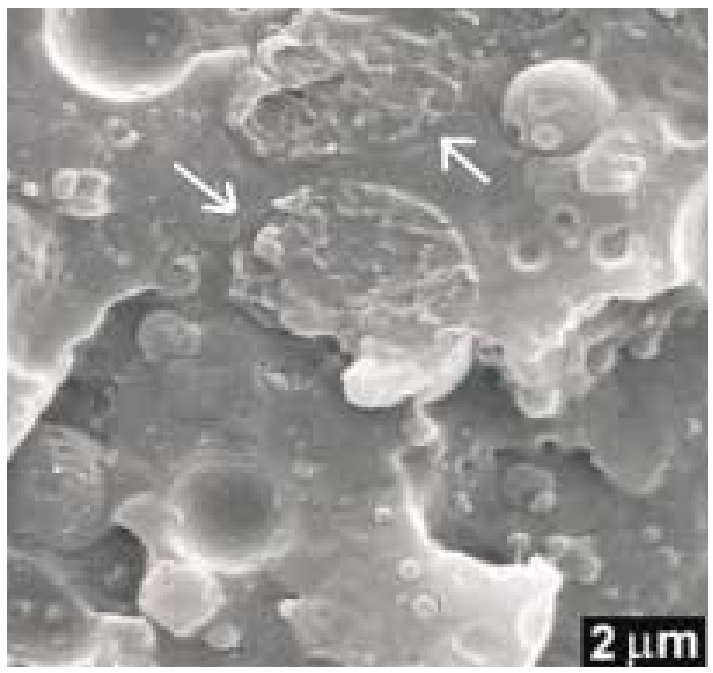

Figura 11. Detalhe da morfologia de uma blenda PP/HIPS (70:30) com 5\% de SEBS. Aumento: $5000 \mathrm{X}$. 
Em síntese, o copolímero SEBS quando adicionado em pequenas quantidades na blenda PP/HIPS, altera a sua morfologia, melhorando a resistência ao impacto.

\section{Agradecimentos}

Os autores agradecem à Polibrasil S.A., EDN S.A e Shell Química, pela concessão dos materiais PP, HIPS e SEBS, respectivamente e à CANDE, pela realização dos ensaios de impacto.

\section{Referências Bibliográficas}

1. Okamoto, K.T.; Denicola, A. J.; Huang, M.C.T. \& Cleuvenbergen, M.V. - "A Synergistic Impact Modifier Combination for Polypropylene/Polystyrene Alloys", in: ANTEC'93, p. 2103-2106 (1993).

2. Melo, T. J. A. ; Brito, K. G. Q. \& Carvalho, L. H. "Effects of Mixing Conditions, Composition and Compatibilizing Agent on Mechanical Properties of PP/HIPS Blends", in: VII International Macromolecular Colloquium, Gramado-RS, set (1996).

3. Fortelny, I.; Michalkova, D. \& Mikesova, J. - J. Appl. Polym. Sci., 59, p.155-164 (1996).

4. Matos, M.E.; Rosales, C.; Muller, A. J. \& Favis, B.D. "Polystyrene / Polypropylene Blends: Morphology / Interphase Relationship, Rheological and Calorimetric Behavior", in: ANTEC'97, p.1-6 (1997).

5. Catálogo Técnico de Borrachas Termoplásticas KRATON® - Shell Química Corporation.

6. Han C. D. "Rheology in Polymer Processing" - Academic Press Inc., New York, (1976).

7. Utracki, L. A . - "Polymer Alloys and Blends: Thermodynamics and Rheology", Hanser Publi., New York, (1989).

8. Paul, D.R. \& Newman, S. - "Polymer Blends", Vols. 1 e 2, Academic Press, New York (1981).

9. Folkes, M.J. \& Hope, P.S. - "Polymer Blends and Alloys", Blackie Academic \& Professional, Glasgow (1995).

10. Plochocki, A. P.; Dagli, S. S. \& Andrews, R. D. Polym. Eng. \& Sci., 30, p.741-752 (1990).

11. Gupta, A. K. \& Purwar, S. N. - J. Appl. Polym. Sci., 29 , p. 1079-1093 (1984).

12. Gupta, A. K. \& Purwar, S. N. - J. Appl. Polym. Sci., 30, p. 1777-11798 (1985).
13. Srinivasan, K.R. \& Gupta, A. K. - J. Appl. Polym. Sci., 53, p. 1-17 (1994).

14. Castro, J.L.D. - "Comportamento Reológico e Mecânico do Sistema PP/ SEBS/ PEBD”, Dissertação de Mestrado, UFSCar, Brasil (1995).

15. Gupta, A. K. \& Purwar, S. N. - J. Appl. Polym. Sci., 30, p. $1799-1814$ (1985).

16. Calumby, R.B.R.; Melo, T.J.A.; Brito, K.G.Q.; Carvalho, L.H. \& Spieth, E. - " The Influence of a SEBS Copolymer as a Compatibiliser for a PP/HIPS Blend" , in: Simposio Argentino de Polimeros, p. 92-93, Bahia Blanca -Argentina, nov (1997).

17. Calumby, R.B.R.; Melo, T.J.A. ; Brito, K.G.Q.; Carvalho, L.H. \& Spieth, E. - "Uso de um Copolímero Tribloco na Compatibilização da Blenda SUPLEN/HIPS", in: Anais do $4^{\circ}$ Congresso Brasileiro de Polímeros, Salvador BA, set/out (1997).

18. Yang, L.Y; Smith, T.G. \& Bigio - "Melt Blending of Linear Low Density Polyethylene and Polystyrene - Effects of Processing Conditions, Composition, and Compatibilizers upon Morphology Development”, in: ANTEC'94, p. 2428-2432 (1994).

19. Bucknall, C.B. - "Toughened Plastics" Applied Science Publishers, London (1977).

20. Lindsey, C. R.; Paul, D.R. \& Barlow J.W. - J. Appl. Polym. Sci, 26, p.1-8 (1981).

21. Park, C.P. \& Cklingerman, G. P. - “ Compatibilizing PE-PS Blends with Ethylene-Styrene Copolymers", in: Plastics Engineering, p.97-99, mar (1997).

22. Nielsen, L.E.- "Mechanical Properties of Polymers and Composites", Vol. 2, p. 347-348, Marcel Dekker, Inc., New York (1974).

23. Bourry, D \& Favis, B. D. - Polymer, 39, p.1851- 1856 (1998).

24. Favis, B.D. - J. Appl. Polym. Sci., 39, p.285-300 (1990).

25. Bordereau, V.; Shi, Z. H.; Utracki, L.A . Sammut, P. \& Carrega, M. - Polym. Eng. \& Sci., 32, p.18461856 (1992).

26. Sundararaj, U. \& Macosko, C. W. - Polym. Eng. \& Sci., 32, p.1814-1823 (1992).

Recebido: 04/06/99

Aprovado: 15/03/00 